\title{
Management of high-grade renal traumas with collecting system injuries
}

Jennifer A. Locke; Sarah Neu; Roshan Navaratnam; Andrea Phillips; Avery B. Nathens; Sender Herschorn; Ronald Kodama

Department of Surgery, Sunnybrook Health Sciences Centre and the University of Toronto, Toronto, ON, Canada

Cite as: Locke JA, Neu S, Navaratnam R, et al. Management of high-grade renal traumas with collecting system injuries. Can Urol Assoc J 2021 May 11; Epub ahead of print. http://dx.doi.org/10.5489/cuaj.7115

Published online May 11, 2021

Correspondence: Dr. Ronald Kodama, Sunnybrook Health Sciences Centre, Toronto, ON, Canada; ron.kodama@sunnybrook.ca

\section{$* * *$}

Abstract

Introduction: Approximately 50\% of all high-grade renal traumas (HGRT, American Association for the Surgery of Trauma [AAST] grade 4/5) have associated collecting system injuries. Although most of these collecting system injuries will heal spontaneously, approximately $20-30 \%$ of these injuries are managed with ureteric stents. The objective of the study was to review the management of HGRT with collecting system injuries in a level 1 trauma center.

Methods: This was a single-center, retrospective cohort study of trauma patients with HGRT and collecting system injuries from 1998-2019.

Results: We identified 147 patients with HGRT. Of the 105 patients who had trauma computed tomography (CT) imaging within 24 hours, 46 were found to have collecting system injuries. Seven of these patients underwent intervention based on initial CT findings; the remaining 39 patients with urinary extravasation were conservatively managed. Of the 37 patients who underwent reimaging, 22 (59\%) demonstrated a stable or resolving collection and $15(41 \%)$ demonstrated continued urinary extravasation. Resolution of extravasation on subsequent imaging was observed in 10 of those patients, while five patients (14\%) required intervention (four stents, one percutaneous drain) for symptoms/signs of urinary extravasation. 
Conclusions: In this study, most patients with HGRT and collecting system injuries did not require intervention unless the patient became symptomatic. The majority of collecting system injuries resolved with no intervention. This study underscores the need for future prospective trials to investigate the necessity of intervening in HGRT collecting system injuries and, secondarily, the need for routine reimaging in these asymptomatic patients.

\section{Introduction}

High-grade renal trauma (HGRT - American Association for the Surgery of TraumaAAST grade $4 / 5^{1}$ ), account for $18-43 \%$ of all renal traumas ${ }^{2-6}$. Current guidelines recommend follow-up imaging at 48 hours in all conservatively managed HGRT to prevent complications associated with a developing urinoma or hemorrhage ${ }^{7-9}$. The guidelines also recommend urinary stenting for urinary extravasation or percutaneous drainage of a urinoma if the patient becomes symptomatic ${ }^{7-9}$. However, it is unclear whether any intervention is necessary in the asymptomatic patient after repeat imaging reveals continued urinary extravasation.

In a systematic review, Keihani et al. found that approximately half of all HGRT AAST grade $4 / 5$ have associated collecting system injuries ${ }^{10}$. Twenty-nine percent of all patients with HGRT and collecting system injuries underwent ureteral stent placement for urinary extravasation ${ }^{10}$. In another multi-institutional series (2014-2017) there were 195 AAST grade $4 / 5$ renal injuries of whom 54 underwent nephrectomy ${ }^{11}$. In this series 29/141 patients (21\%) with HGRT, who did not undergo nephrectomy, had ureteric stents placed for urinary extravasation ${ }^{11}$. However, the indication for stent placement in these collecting system injuries was not specified. As routine reimaging is recommended in the renal trauma guidelines at 48 hours ${ }^{7-9}$, Bayne et al. evaluated whether reimaging changed management in a series of 216 HGRT patients ${ }^{12}$. Of 48 asymptomatic patients who underwent routine image restaging (average 3.55 days; range $0.44-28.9$ days), the only patients requiring intervention were patients with previously identified urinary extravasation. Of these 24 patients who had continued urinary extravasation on repeat imaging, $6(25 \%)$ underwent ureteral stent insertion ${ }^{12}$. However, the indications for stent placement were not defined and likely left to the attending urologists' discretion ${ }^{12}$. They concluded that reimaging in all asymptomatic HGRT (AAST grade 4/5) patients may not be necessary and possibly should be performed in only those with collecting system injuries. 
The objective of this study was to review the management of HGRT with collecting system injuries in a level 1 trauma centre. 


\section{Methods}

Ethics was approved through the Sunnybrook Health Sciences Centre institutional review board (\#2582). We conducted a retrospective chart review of all AAST grade 4/5 renal traumas identified at a level one trauma centre between 1998-2019. All renal traumas were identified through the institutional trauma database. Specifically, grade $4 / 5$ renal traumas were identified based on CT scan, operative or pathology reports or chart notes (AAST criteria) reviewed by the trauma data analyst and assigned abbreviated injury score codes ${ }^{1}$. Grade 1-3 renal traumas were excluded from this study. Two urologists identified those Grade 4/5 renal traumas with collecting system injuries by reviewing the initial trauma CT scans and reports. Trauma CT imaging evolved over the time period of the study. The current trauma protocol includes a non-contrast head scan followed by a pump bolus of contrast ( $4 \mathrm{ml} / \mathrm{sec}$ for $25-30$ seconds). Once the bolus is administered there is a scan of the neck to upper abdomen in the arterial phase and the mid-chest to pelvis in the late arterial/early portal venous phases. The duration of the scan is less than 70 seconds. If a grade 3-5 renal injury is identified a delayed excretory phase is done at 10 minutes. Data collection included initial and follow-up imaging modality, timing of reimaging, radiologic interpretation, as well as subsequent interventions and outcomes. Descriptive statistical analysis was utilized to analyze the data.

\section{Results}

\section{Population demographics (Table 1)}

One-hundred-forty-seven patients with HGRT (AAST Grade 4/5) were identified between 1998-2019) of 1267 patients with a kidney injury of any grade (12\%) (Figure 1). The majority of patients were male $(80 \%)$. The average age of the patients was 35 years with a median age of 30 (range 18-83). Seventy-one percent experienced blunt trauma while the remainder experienced penetrating trauma.

Of these 147 patients, 42 (29\%) did not have immediate CT imaging because of hemodynamic instability. One patient expired in the trauma bay, and the remaining patients went directly to the operating room. Thirty-three of these patients $(80 \%)$ underwent a nephrectomy and the remaining had either a partial nephrectomy ( 5 patients), renorrhaphy (1 patient) or a retroperitoneal drain (1 patient). One patient died in the operating room after having clinical findings of a Grade 4/5 renal trauma. Penetrating injuries accounted for 28/41 (68\%) patients who went directly to the operating room. Penetrating trauma accounted for 19 nephrectomies and all partial nephrectomies, renorrhaphy and drain placement. The majority of nephrectomies (31/33) were performed by trauma surgeons. Two of the partial nephrectomies and the renorrhaphy were performed by a urologist. 
The remaining 105 (71\%) patients with HGRT, had their initial CT trauma imaging within 24 hours of injury. Fifty-nine of these patients had no collecting system injuries and were managed with no intervention (30), renal embolization (13), nephrectomy (13) and open renal repair (3). Nineteen patients underwent a trauma laparotomy for other injuries without renal exploration.

\section{Diagnosis and management of collecting system injuries}

Of the 46 with a collecting system injury, 36 (78\%) were due to blunt trauma and the remainder were penetrating. Seven patients underwent immediate intervention based on their initial CT findings. One patient had a stent inserted for a solitary kidney, 3 patients had retroperitoneal kidney drains placed at the time of laparotomy, 2 patients underwent a nephrectomy and 1 patient underwent surgical exploration, the details of which are unknown.

The remainder of the patients $(39 / 46 ; 85 \%)$ were managed conservatively and did not undergo any renal intervention based on their initial CT scan (Figure 2). Nine underwent a trauma laparotomy for other concomitant injuries without exploration of the kidney. Two did not have reimaging while in hospital for unknown reasons. Thirty-seven patients underwent repeat imaging (abdominal U/S 8, non-contrast CT 3 and contrast CT 26). There was no set reimaging protocol in these cases. Of those reimaged, $22(59 \%)$ demonstrated a stable or resolving collection (i.e. smaller or non-expanding collection on $\mathrm{U} / \mathrm{S}$ or no extravasation with co-existing smaller or non-expanding collection on CT (median re-imaging time 3 days (range 1-112 days)) and 15 (41\%) demonstrated continued urinary leak (median re-imaging time 4 days (range 1-28 days)). The 22 patients with stable or resolving collections remained asymptomatic and required no follow-up intervention. Resolution of urinary leak on subsequent imaging was observed in 10 of the 15 patients (median follow-up 6 weeks (range 4-6 weeks) while 5 patients (14\%) required subsequent intervention (4 stents, 1 drain) for symptoms or ongoing urinary leak. Of the 5 patients who required intervention patient 1 had a horseshoe kidney with significant injury to the isthmus and bilateral collecting system injuries with increasing abdominal pain. Patient 2 developed worsening abdominal and flank pain secondary to an increasing urinoma. Patient 3 developed a fever and abdominal distention 9 days after HGRT due to a gunshot wound and was found to have a small urine leak. A fourth asymptomatic patient had an ureteropelvic junction (UPJ) leak and was stented based on the diagnosis of persistent leak on reimaging CT. The fifth patient became symptomatic 13 days after the trauma and was found to have a large urinoma. In these 5 patients we were unable to determine if mechanism of injury was a predictor for delayed intervention. 


\section{Urologist involvement in trauma care}

Overall, 57/105 or 54\% of those with immediate initial CT trauma imaging had a urologist involved in their care (Table 1). Urological involvement increased over the duration of the study (first quartile 1998-2002 32\% to last quartile 2013-2019 86\%). Thirty-five of the forty-six patients $(76 \%)$ with collecting system injuries had a urologist involved in their care.

\section{Discussion}

Current AUA, EAU and WSES-AAST guidelines suggest reimaging routinely at 48 hours in patients with HGRT regardless of symptoms ${ }^{7-9}$. The AUA urotrauma guidelines state that, "follow-up CT imaging (after 48 hours) is prudent in patients with deep renal injuries because these are prone to developing troublesome complications such as urinoma or hemorrhage." 9 EAU guidelines state that, "Repeat imaging two-four days after trauma minimizes the risk of missed complications, especially in grade 3-5 blunt injuries." 7 WSES-AAST guidelines state that, "in severe injuries (AAST IV-V), contrast-enhanced CT scan with excretory phase (in cases with possible or documented urinary extravasation) or ultrasound and contrast-enhanced US are suggested within the first 48 hours after trauma in adult patients." 8

In this study, of the 37 conservatively managed patients who had HGRT with collecting system injuries and had various modalities of repeat imaging only $5(14 \%)$ had interventions on repeat imaging. Four out of the 5 patients required intervention because of symptoms related to their collecting system injury and 1 was asymptomatic but stented because of a solitary kidney. All 5 patients had satisfactory outcomes in follow-up. The remaining $86 \%$ of those reimaged were asymptomatic, did not require any additional intervention and demonstrated a stable or resolving collection on subsequent reimaging. This data suggests that the majority of asymptomatic patients with HGRT and collecting system injuries will resolve with time and reimaging should be reserved for only symptomatic patients. It is unclear in the literature which imaging modality (CT versus $\mathrm{U} / \mathrm{S}$ ) is the best choice for reimaging in asymptomatic patients. In this study there was no reimaging protocol so both modalities were used. An ultrasound may detect an enlarging leak by measuring increased collection size.

Routine repeated CT imaging is not without the possibility of increased long term cancer risk in these polytrauma patients ${ }^{13,14}$. It has been shown that trauma patients are exposed to significant amounts of CT radiation during their time in hospital ${ }^{13,15}$. The "as low as reasonably achievable" (ALARA) principle was introduced in 2008 to emphasize utilizing the lowest dose of radiation possible and reduce unnecessary imaging 16. Several groups are trying to reduce ionizing radiation exposure in trauma patients in keeping with the ALARA principle ${ }^{17,18}$. In keeping with this principle, polytrauma 
patients who require reimaging for other injuries, it seems prudent that the urology team be aware of these investigations and suggest appropriate genitourinary scan protocols if required at the same time. This will reduce overall unnecessary repeat $\mathrm{CT}$ scans and radiation exposure.

A surprise finding in this study, was that overall, only 54\% of the HGRT patients with CT imaging had a urologist involved in their care. This did improve from $32 \%$ (1998-2002) to 86\% (2013-2019) through the study time period. Specific to those with collecting system injuries $76 \%$ had a urologist involved in their care. Of the 46 collecting system injuries 11 did not have a urologist involved in their care; 9 of these cases occurred before 2007. These trends highlight how the trauma surgeons are more frequently involving the urologist in the care of modern day HGRTs. In a review by Yeung and Brandes it was found that trauma surgeons were more likely to want urologists to immediately stent collecting system injuries compared to urologists (50 vs $24 \%, \mathrm{p}<0.001)^{19}$. Furthermore, the authors found differing opinions in treating renal traumas between trauma surgeons and urologists and recommended large-scale, multiinstitutional, interdisciplinary prospective studies on renal trauma to "standardize" management ${ }^{19}$.

There were several limitations to this study. It is a single centre, small number, retrospective review over a long period of time (1998-2019). In this study, we did not systemically evaluate the severity of collecting system injuries other than AAST grading and thus cannot make any recommendation on which patients should have reimaging or intervention. There was no standardized method for managing collecting system injuries among the urologists but in general a more conservative approach to these injuries was undertaken. Lastly, it is unknown if earlier intervention in those who ultimately required it would have prevented the onset of delayed symptoms. Also as most patients did not require intervention, we cannot comment if early stent/drain placement in those that were asymptomatic would have led to unnecessary morbidity. Urologist involvement was only determined based on chart review and therefore some consultations may not have been recorded.

\section{Conclusions}

In this study the majority of patients with HGRT and collecting system injuries did not require an intervention unless the patient became symptomatic. The majority of collecting system injuries resolved with no intervention. This study underscores the need for future prospective trials to investigate the necessity of intervening in HGRT collecting system injuries and secondarily, the need for routine reimaging in the asymptomatic HGRT. 


\section{References}

1. Moore EE, Shackford SR, Pachter HL, et al. Organ injury scaling: spleen, liver, and kidney. J Trauma. Dec 1989;29(12):1664-6.

2. Mann U, Zemp L, Rourke KF. Contemporary management of renal trauma in Canada: A 10-year experience at a level 1 trauma centre. Can Urol Assoc J. Jun 2019;13(6):E177-E182. doi:10.5489/cuaj.5581

3. Voelzke BB, Leddy L. The epidemiology of renal trauma. Transl Androl Urol. Jun 2014;3(2):143-9. doi:10.3978/j.issn.2223-4683.2014.04.11

4. Pillay V, Pillay M, Hardcastle TC. Renal trauma in a Trauma Intensive Care Unit population. S Afr J Surg. Dec 2019;57(4):29-32.

5. Salem MS, Urry RJ, Kong VY, Clarke DL, Bruce J, Laing GL. Traumatic renal injury: Five-year experience at a major trauma centre in South Africa. Injury. Jan 2020;51(1):39-44. doi:10.1016/j.injury.2019.10.034

6. Khoschnau S, Jabbour G, Al-Hassani A, et al. Traumatic Kidney Injury: An Observational Descriptive Study. Urol Int. 2020;104(1-2):148-155. doi:10.1159/000504895

7. Serafetinides E, Kitrey ND, Djakovic N, et al. Review of the current management of upper urinary tract injuries by the EAU Trauma Guidelines Panel. Eur Urol. May 2015;67(5):930-6. doi:10.1016/j.eururo.2014.12.034

8. Coccolini F, Moore EE, Kluger Y, et al. Kidney and uro-trauma: WSES-AAST guidelines. World J Emerg Surg. 2019;14:54. doi:10.1186/s13017-019-0274-X

9. Morey AF, Brandes S, Dugi DD, et al. Urotrauma: AUA guideline. J Urol. Aug 2014;192(2):327-35. doi:10.1016/j.juro.2014.05.004

10. Keihani S, Anderson RE, Fiander M, et al. Incidence of urinary extravasation and rate of ureteral stenting after high-grade renal trauma in adults: a meta-analysis. Transl Androl Urol. May 2018;7(Suppl 2):S169-S178. doi:10.21037/tau.2018.04.13

11. Keihani S, Xu Y, Presson AP, et al. Contemporary management of high-grade renal trauma: Results from the American Association for the Surgery of Trauma Genitourinary Trauma study. J Trauma Acute Care Surg. 03 2018;84(3):418-425. doi:10.1097/TA.0000000000001796

12. Bayne DB, Tresh A, Baradaran N, et al. Does routine repeat imaging change management in high-grade renal trauma? Results from three level 1 trauma centers. World J Urol. Jul 2019;37(7):1455-1459. doi:10.1007/s00345-018-25132

13. Beatty L, Furey E, Daniels C, Berman A, Tallon JM. Radiation Exposure From CT Scanning in the Resuscitative Phase of Trauma Care: A Level One Trauma Centre Experience. CJEM. Nov 2015;17(6):617-23. doi:10.1017/cem.2014.61

14. Howard A, West R, Iball G, Panteli M, Pandit H, Giannoudis PV. An Estimation of Lifetime Fatal Carcinogenesis Risk Attributable to Radiation Exposure in the First Year Following Polytrauma: A Major Trauma Center's Experience Over 10 
Years. J Bone Joint Surg Am. Aug 2019;101(15):1375-1380. doi:10.2106/JBJS.18.01334

15. Kritsaneepaiboon S, Jutiyon A, Krisanachinda A. Cumulative radiation exposure and estimated lifetime cancer risk in multiple-injury adult patients undergoing repeated or multiple CTs. Eur J Trauma Emerg Surg. Feb 2018;44(1):19-27. doi:10.1007/s00068-016-0665-6

16. Musolino SV, DeFranco J, Schlueck R. The ALARA principle in the context of a radiological or nuclear emergency. Health Phys. Feb 2008;94(2):109-11. doi:10.1097/01.HP.0000285801.87304.3f

17. Kahn J, Grupp U, Kaul D, Böning G, Lindner T, Streitparth F. Computed tomography in trauma patients using iterative reconstruction: reducing radiation exposure without loss of image quality. Acta Radiol. Mar 2016;57(3):362-9. doi: $10.1177 / 0284185115580839$

18. Kalra MK, Rizzo SM, Novelline RA. Reducing radiation dose in emergency computed tomography with automatic exposure control techniques. Emerg Radiol. Jul 2005;11(5):267-74. doi:10.1007/s10140-004-0395-7

19. Yeung LL, Brandes SB. Contemporary management of renal trauma: differences between urologists and trauma surgeons. J Trauma Acute Care Surg. Jan 2012;72(1):68-75; discussion 75-7. doi:10.1097/TA.0b013e31823e29f6 
Figures and Tables

Fig. 1. Management of 147 patients with high-grade renal traumas (HGRTs). CT: computed tomography; GSW with lap: gunshot wound with laparotomy; OR: operating room.

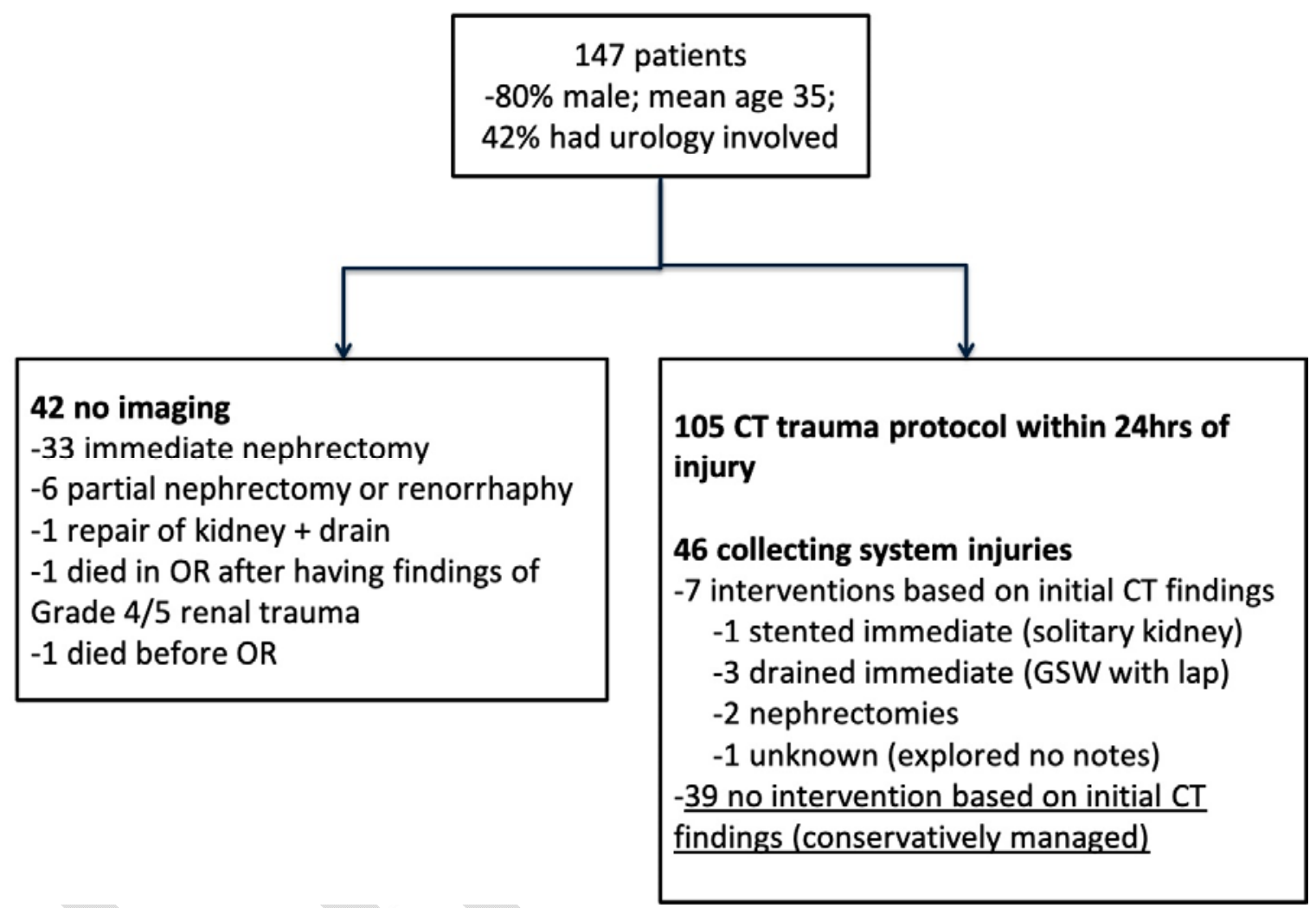


Fig. 2. Management of 39 patients with high-grade renal traumas (HGRT) and collecting system injuries who, based on initial CT findings, were conservatively managed. CT con: computed tomography contrast; CT non con: computed tomography non-contrast: U/S: ultrasound.

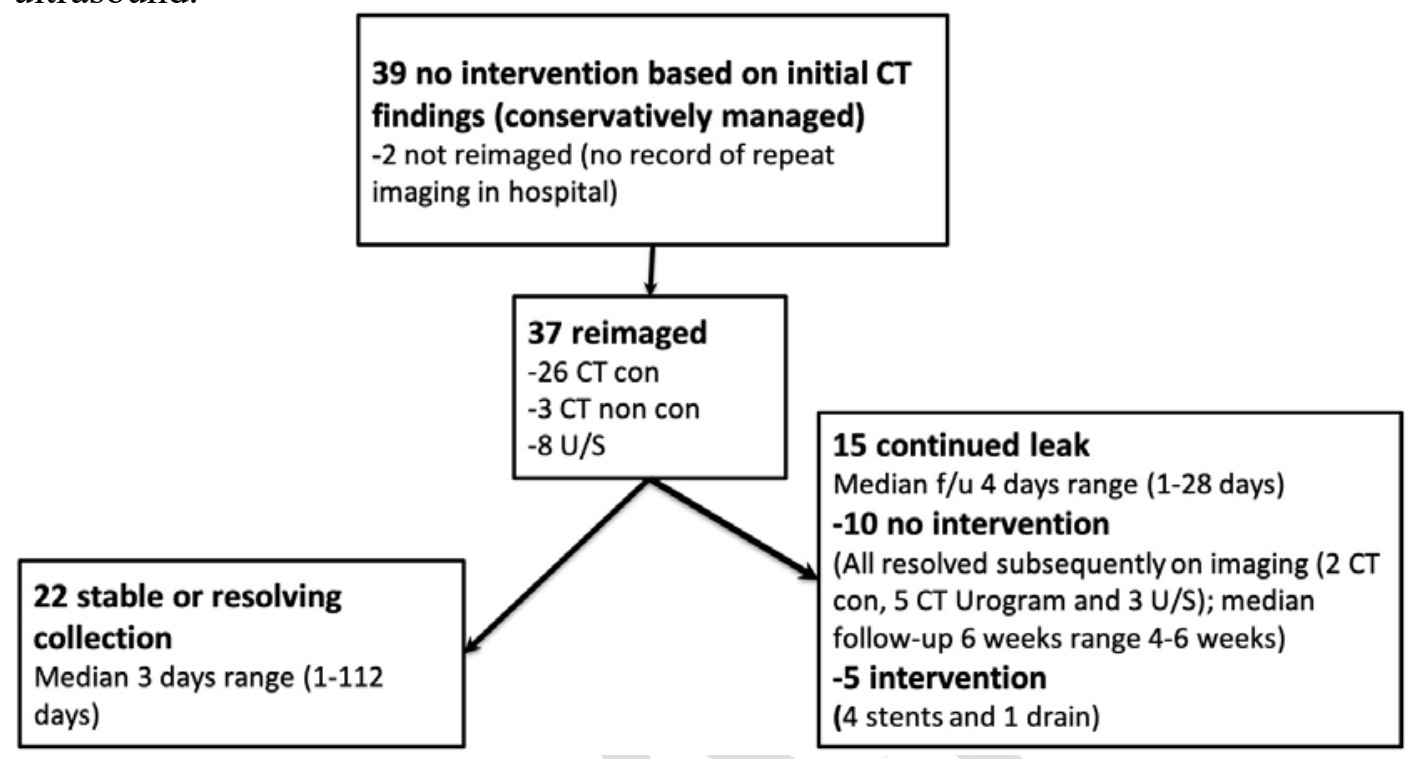

\begin{tabular}{|l|c|}
\hline \multicolumn{2}{|l|}{ Table 1. Demographics of patients with high-grade renal traumas } \\
\hline Variable & Total \\
\hline Male, $\mathrm{n}(\%)$ & $118(80)$ \\
Female, $\mathrm{n}(\%)$ & $29(20)$ \\
\hline Age & 35.2 \\
Mean (years) & $30(18-83)$ \\
Median (range) (years) & $105(71)$ \\
\hline Mechanism of renal trauma & $42(29)$ \\
Blunt (motor vehicle collision, falls, work site, etc.), n (\%) & $22(52)$ \\
Penetrating, n (\%) & $57(54)$ \\
Penetrating due to gunshot injury, n (\%) & $6 / 19(32)$ \\
\hline Patients with immediate CT trauma protocol imaging with a & $9 / 34(27)$ \\
urologist involved in care, $\mathrm{n}(\%)$ & $17 / 23(74)$ \\
1998-2002, n (\%) & $25 / 29(86)$ \\
2003-2007, n (\%) & \\
2008-2012, n (\%) & 38.2 \\
2013-2019, n (\%) & 34.4 \\
\hline Injury severity score & \\
Before 2008 (mean) & \\
After 2008 (mean) & \\
\hline
\end{tabular}

CT: computed tomography. 